\title{
Proteoglycans in Albo-Papuloid Lesions of the Pasini Form of Dominant Dystrophic Epidermolysis Bullosa
}

\author{
HIROSHI HACHISUKA, NOBUHIRO YAMAMOTO, HIDEKI SAKIHAMA \\ AND YOICHIRO SASAI \\ Department of Dermatology, Kurume University School of \\ Medicine, Kurume, 830 Japan
}

Received for publication December 5, 1994

\begin{abstract}
Summary: Epidermolysis bullosa is a group of inherited blistering diseases classified into three main sub-groups on the basis of the level of cleavage within the skin. In dominant dystrophic epidermolysis bullosa, characterized by cleavage below the basal lamina, two variants can be distinguished by the presence (Pasini form) or absence (Cockayne-Touraine form) of albo-papuloid lesions. The present study was undertaken to investigate the glycosaminoglycan chains of proteoglycans in the albopapuloid lesions of a patient with the Pasini form, using histochemical and immunohistochemical methods. Histological examination revealed no dermo-epidermal separation. In the dermis, the papillary and subpapillary layers were slightly homogeneous, and exhibited a strong affinity towards alcian blue, which was abolished by treatment with chondroitinase ABC or in the presence of $\mathrm{MgCl}_{2} 0.3 \mathrm{M}$, but was resistant to digestion with streptomyces hyaluronidase. The papillary and subpapillary layers were intensely stained with a monoclonal antibody against small size proteoglycan with dermatan sulfate. These results suggest the presence of degraded dermatan sulfate proteoglycan in the papillary and subpapillary dermis of albo-papuloid lesions in the Pasini form of dystrophic epidermolysis bullosa.
\end{abstract}

Key words: epidermolysis bullosa - blister — proteoglycan - dermatan sulfate - hyaluronic acid

\section{Introduction}

Epidermolysis bullosa (EB) is a group of inherited disorders characterized by severe blistering after minor trauma. Three main subgroups are distinguished on the basis of the level of cleavage within the skin: (1) epidermolysis bullosa simplex; (2) junctional epidermolysis bullosa; and (3) dystrophic epidermolysis bullosa (Fine et al. 1991).

In dystrophic epidermolysis bullosa
(DEB), cleavage occurs below the basal lamina and the blisters heal, leaving a scar. Six subtypes of DEB have been identified, two of which are inherited in a dominant mode and four in a recessive one. The two subtypes of dominantly inherited dystrophic epidermolysis bullosa (D-DEB) are distinguished by the presence (Pasini form) or absence (Cockayne-Touraine form) of albopapuloid lesions. These lesions appear on the lower back of the patients in 
adolescence. A previous study by us showed an increased amount of degraded chondroitin sulfates in the skin of a patient with the Pasini form of D-DEB (P-D-DEB) (Sasai et al. 1973). Furthermore, the urinary excretion of both native and partially degraded dermatan sulfate was increased in the patient (Endo et al. 1974). Subsequently, Bauer et al. (1979) reported that fibroblasts from nonlesional skin tissues of the patients with P-D-DEB synthesize and release excessive amounts of sulfated glycosaminoglycans (GAGs) as compared with fibroblasts from patients with other forms of $\mathrm{EB}$, and postulated that the in vitro abnormality in GAG metabolism could be used as a diagnostic tool for this form of EB.

Proteoglycans (PGs) are major components of the extracellular matrix. They comprise a large family of molecules, which differ in the chemical nature of their core protein and in their GAG chains. It has been difficult to determine histochemically the localization of heterogeneous PG species. The recent development of monoclonal antibodies to PGs has made possible to determine the precise localization of PGs in various human tissues. The present study was undertaken to study GAG chains of PG in the skin of a patient with P-D-DEB by means of immunohistochemical methods.

\section{Materials and Methods}

Biopsy skin specimens were taken from papules on the back of a patient with P-D-DEB. As physiological controls, we took specimens from the back of agematched healthy women. The specimens were fixed in $1 \%$ acetic acid in $95 \%$ ethanol for $24 \mathrm{hs}$ at $4{ }^{\circ} \mathrm{C}$. Following fixation, the specimens were dehydrated, cleared and embedded in paraffin wax. Sections were cut with a microtome at a thickness of $6 \mu \mathrm{m}$, affixed on slides, deparaffinized in xylene and rehydrated in a series of descending concentrations of ethanol. In addition to routine staining methods, the tissue sections thus treated were subjected to histochemistry, immunohistochemistry and enzyme digestion as described below.

\section{Histochemistry}

Alcian blue staining: Alcian blue was used as a $0.5 \%$ solution in $0.05 \mathrm{M}$ acetate buffer at $\mathrm{pH} 5.8$ (Scott and Dorling, 1965). The solution was divided into aliquots to which $\mathrm{MgCl}_{2}$ was added, so as to produce molarities ranging from 0.025 to $2.0 \mathrm{M}$. Sections were stained for $12 \mathrm{hs}$ and rinsed three times for $5 \mathrm{~min}$ each in buffer of the same concentration as that used for the staining. After rinsing, sections were washed with distilled water three times for $3 \mathrm{~min}$ each, dehydrated in ethanol, cleared in xylene and mounted in Permount.

In certain sections, $1.0 \%$ Alcian blue solution was prepared in $3.0 \%$ acetic acid. Rehydrated sections were stained in this solution for $30 \mathrm{~min}$, and rinsed in $3.0 \%$ acetic acid prior to washing in distilled water (Lillie, 1965).

Periodic acid-Schiff (PAS) (Lillie, 1965): Hydrated sections were soaked in $50 \mathrm{mM}$ periodic acid for $10 \mathrm{~min}$, washed in running tap water for $10 \mathrm{~min}$, and stained with Schiff's reagent.

\section{Immunohistochemistry}

Antibodies: Monoclonal antibodies used 
were: 2B1 (against chondroitin sulfate large PG, which was purified from a yolk sac tumor) (Sobue et al. 1989), 3B3 (against a stub of $\triangle$ di-6S unit binding to core protein obtained by treating chondroitin sulfate PG with chondroitinase ABC)(Couchman et al. 1984), 6B6 (against small PG with dermatan sulfate and chondroitin sulfate, which was purified from the capsular tissue of human ovarian fibroma) (Sobue et al. 1988), and CS56 (which reacts with chondroitin sulfate but not with dermatan sulfate)(Avnur and Geiger, 1984). These antibodies were obtained from Seikagaku Kogyo Co., Tokyo, Japan. In addition, biotinylated hyaluronic acid-binding protein (HABP) was prepared as described by Tengblad (1979).

Staining procedure: Deparaffinized tissue sections were soaked in methanol containing $0.3 \% \mathrm{H}_{2} \mathrm{O}_{2}$, to inhibit the following staining procedures for the detection of antigens. For the staining of antigens for 2B1 and CS56, sections were pretreated with trypsin. For the staining of antigens for $3 \mathrm{~B} 3$ and $6 \mathrm{~B} 6$, sections were pretreated with chondroitinase ABC. Sections treated and untreated with the enzyme were allowed to react with normal goat serum (1:100 dilution) and then reacted with diluted culture fluid (1:1000-5000) containing the monoclonal antibody. HABP was diluted 1:100. After $1 \mathrm{~h}$-reaction with the monoclonal antibody at room temperature, excess antibody was removed by washing the sections with PBS. The tissue-bound antibodies were labeled with biotinylated anti-mouse immunoglobulin and reacted with peroxidaseconjugated streptoavidin. After rewashing with PBS, tissue sections were soaked in $0.05 \mathrm{M}$ acetate buffer ( $\mathrm{pH} 5.0$ ) containing 0.02\% 3-amino-9-ethyl-carbazole and $0.009 \% \mathrm{H}_{2} \mathrm{O}_{2}$, allowed to react for 5$10 \mathrm{~min}$, and mounted in glycerin jelly. In negative controls, primary antibodies were replaced by normal mouse serum for the reaction. For identifying hyaluronic acid, sections were treated with streptomyces hyaluronidase prior to the staining.

\section{Enzyme digestions}

Trypsin digestion (Avnur and Geiger, 1984): Sections were treated for $20 \mathrm{~min}$ at $37^{\circ} \mathrm{C}$ with trypsin dissolved in PBS at a concentration of $10 \mu \mathrm{g} / \mathrm{ml}$.

Chondroitinase $A B C$ digestion (Sobue et al. 1988): Sections were treated for $2 \mathrm{hs}$ at $37^{\circ} \mathrm{C}$ in chondroitinase ABC dissolved in $0.025 \mathrm{M}$ Tris- $\mathrm{HCl}$ buffer of $\mathrm{pH}$ 8.0 , containing $20 \mathrm{mM}$ sodium acetate and protease inhibitors (10 mM EDTA, $10 \mathrm{mM}$ phenylmethylsulfonylfluoride, and $0.036 \mathrm{mM}$ pepstatin), at a concentration of 0.2 units $/ \mathrm{ml}$.

Streptomyces hyaluronidase digestion (Sasai, 1971b): Sections were treated for $1 \mathrm{~h}$ at $40^{\circ} \mathrm{C}$ in streptomyces hyaluronidase dissolved in $0.02 \mathrm{M}$ acetate buffer of $\mathrm{pH}$ 6.0 at a concentration of $30 \mathrm{TRU} / \mathrm{ml}$.

Testicular hyaluronidase digestion (Sasai, 1971b): Sections were treated for $2 \mathrm{hs}$ at $37^{\circ} \mathrm{C}$ in testicular hyaluronidase dissolved in $0.1 \mathrm{M}$ phosphate buffer of $\mathrm{pH} 6.5$ at a concentration of $0.5 \mathrm{mg} / \mathrm{ml}$. Neuraminidase digestion (Culling et al. 1974): Sections were treated for $18 \mathrm{hs}$ at $37^{\circ} \mathrm{C}$ with neuraminidase (Vibrio cholerae) dissolved in $0.1 \mathrm{M}$ phosphate buffer of $\mathrm{pH} 6.0$ at a concentration of $0.5 \mathrm{mg} / \mathrm{ml}$.

Trypsin, chondroitinase ABC, streptomyces hyaluronidase and testicular hyaluronidase were obtained from 
Seikagaku Kogyo Co., Tokyo, Japan, and neuraminidase was obtained from Calbiochem-Behringer Co., La Jolla, U.S.A.

\section{Results}

\section{Histology}

The skin tissue specimens from the albo-papuloid lesions exhibited a slight hyperkeratosis and focal follicular plugging of the epidermis. The epidermis was somewhat decreased in thickness, but not flattened. In the tissues, no dermoepidermal separation was found. The epidermal basement membrane was well stained either with PAS or silver impregnation method. In the dermis, both the papillary and subpapillary layers were slightly homogeneous (Fig. 1), but no abnormality was detected with any of the connective tissue staining methods used. In the papillary and subpapillary layers elastic fibers were smaller in amount than elsewhere; these fibers were fine and ran parallel to the surface of the skin.

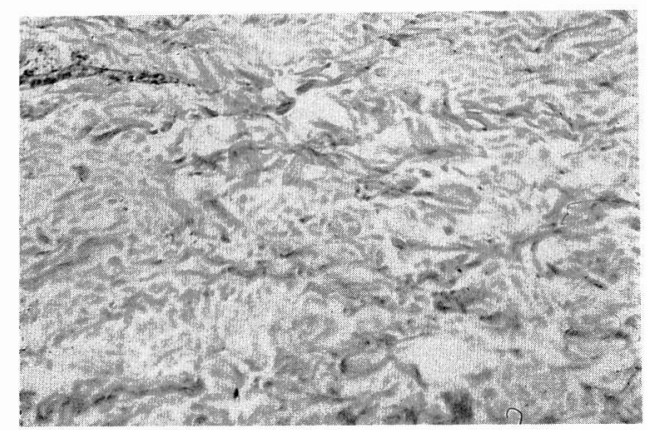

Fig. 1. Upper dermis from albo-papuloid lesion. Slight homogenity is noted in the dermis. Hematoxylin-eosin stain. $\times 120$.

\section{Histochemistry}

In the skin of the patient, the papillary and subpapillary layers showed a strong affinity towards alcian blue, in particular in the interfibrillar spaces. This alcianophilia was completely abolished by prior treatment with chondroitinase $\mathrm{ABC}$, and partially by prior testicular hyaluronidase digestion. However, it was resistant to digestion with streptomyces hyaluronidase or neuraminidase. At $\mathrm{pH} 5.8$, the affinity towards alcian blue was slightly reduced in intensity in presence of $0.15 \mathrm{M}$ magnesium chloride (Fig. 2) and more markedly in presence of $0.25 \mathrm{M}$ of the salt. With the PAS staining procedure, the papillary and subpapillary layers were stained diffusely. The PAS staining was hardly affected by any enzyme digestions used.

\section{Immunohistochemistry}

In the skin of the patient, HABP was found to bind throughout the whole dermis. In the papillary and subpapillary layers the binding was relatively more marked around the collagen fibers (Fig. 3).

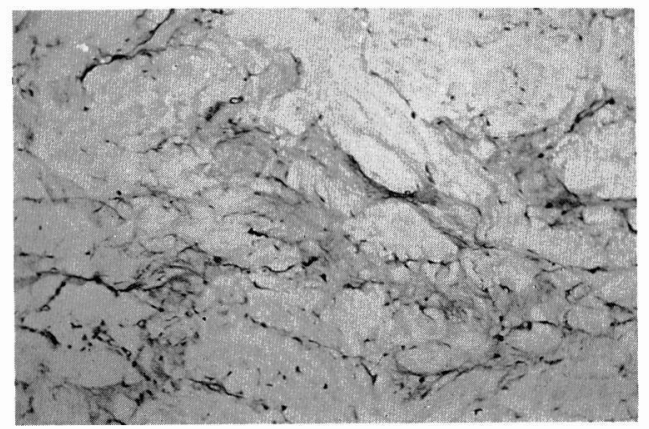

Fig. 2. Upper dermis from albo-papuloid lesion. A strong affinity towards alcian blue is seen, in particular in the interfibrous areas. Alcian blue stain in the presence of $\mathrm{MgCl}_{2} 0.1 \mathrm{M} . \times 120$. 


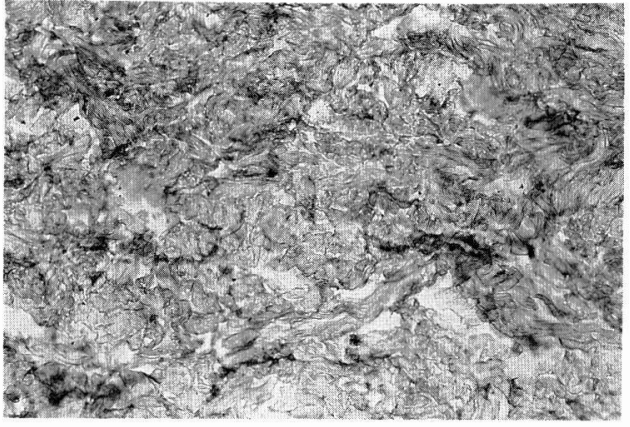

Fig. 3. Upper dermis from albo-papuloid lesion. The dermal tissues around the collagen bind intensely to hyaluronic acid binding protein (HABP). HABP-immunostained $\times 120$.

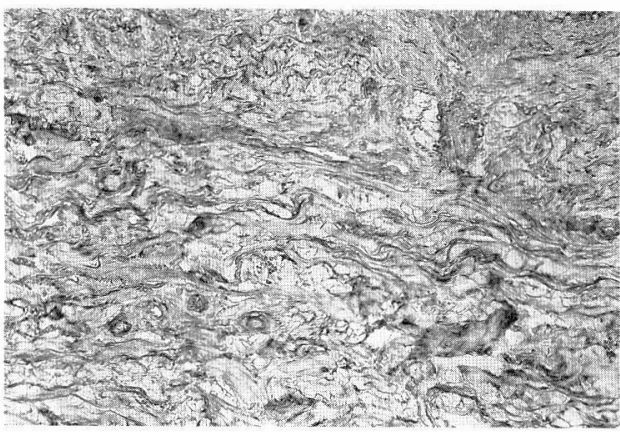

Fig. 4. Upper dermis from albo-papuloid lesion. The dermal tissue is immunoreactive to $6 \mathrm{~B} 6$, monoclonal antibody against small sized proteoglycan with dermatan sulfate and chondroitin sulfate. 6B6-immunostained. $\times 120$.

Such a binding was completely abolished by digestion with streptomyces hyaluronidase. Staining with 2B1 or 6B6 was visualized throughout the dermis, particularly in the papillary and subpapillary dermis (Fig. 4). This staining was not affected in intensity by streptomyces hyaluronidase digestion. Similarly CS56 was bound throughout the dermis, whereas no binding to $3 \mathrm{~B} 3$ was noted.

\section{Discussion}

PGs are large, complex macromolecules distributed ubiquitously in the human body and are localized in the matrices of both mineralizing and nonmineralizing tissues. They consist of GAG chains made of repeating disaccharide units, covalently linked to a core protein. Five main types of GAG chains are known to occur in mammalian connective tissues; hyaluronic acid, heparin/heparan sulfate, chondroitin sulfate, dermatan sulfate and keratan sulfate.

In general, GAGs show a strong affinity towards basic dyes. Each acidic group of GAGs responsible for the affinity can be characterized by its $\mathrm{pK}$ value. At higher $\mathrm{pH}$ values, carboxyl, phosphate, and sulfate groups are all dissociated and ionized. On the other hand, all types of polyanions are precipitated by alcian blue, and waterinsoluble polyanion-dye complexes thus formed can be rendered soluble by the presence of electrolytes, if their concentration is not below a certain critical level (principle of critical electrolyte concentration method by Scott and Dorling, 1965). According to a model experiment, hyaluronic acid reveals an affinity towards alcian blue in the presence of magnesium chloride at $0.1 \mathrm{M}$, dermatan sulfate and chondroitin sulfates $\mathrm{A}$ and $\mathrm{C}$ at $0.7 \mathrm{M}$. The pattern of the critical electrolyte concentration of each GAG in unfixed tissue sections is similar to that in the model experiment, even though the former is nearly 30\% higher than the latter (Sasai, 1971a). In the present work, therefore, the strong affinity towards alcian blue in the upper dermis could be interpreted as implying 
an increased amount of hyaluronic acid. However, the alcianophilia was not affected by treatment with streptomyces hyaluronidase, whereas it was abolished completely by treatment with chondroitinase ABC. Streptomyces hyaluronidase is known to degrade hyaluronic acid only, and chondroitinase $\mathrm{ABC}$ to degrade dermatan sulfate, chondroitin sulfates A and $\mathrm{C}$, and hyaluronic acid. In a previous paper, the data were interpreted as due to an increase in amount of degraded dermatan sulfate, since this was found to be doubled in patient's urine compared with normal controls (Sasai et al. 1973). However, it has been hardly possible to determine the histochemical localization of heterogeneous PG species depending upon their chemical nature. Recently, mono- or polyclonal antibodies raised against PGs have been developed, and a number of investigators have made attempts to detect PGs by means of immunohistochemical methods using antibodies that recognize the different chemical nature of their core proteins or their isomeric GAG chains (Avnur and Geiger, 1984; Couchman et al. 1984; Voss et al. 1985; Poole et al. 1986; Sobue et al. 1988, 1989). Incidentally, hyaluronic acid is not synthesized in the form $P G$, and the highly regular and conserved nature of PG, and in particular the highly regular and conserved nature of hyaluronic acid molecule could apparently be responsible for the failure in attempts to produce antibodies to undergraded hyaluronic acid by means of conventional techniques (Hascall and Hascall, 1981). Hyaluronic acid interacts with one end of the PG core protein, the hyaluronic acid-binding region, in a highly specific manner. Such an inter- action is stabilized by a link protein that occurs in two closely related forms and binds to hyaluronic acid on the one hand and the hyaluronic acid-binding region of the PG on the other. Based upon these data, Ripellino et al. (1985) have developed a specific and sensitive method to visualize the localization of hyaluronic acid in a variety of tissues. In the present study, HABP was prepared as described by them. However, any significant difference could be recognized in HABP binding between the patient's and normal control dermis. When $2 \mathrm{~B} 1$ or $6 \mathrm{~B} 6$ was applied, the upper dermis of the patient's skin exhibited a strong binding to such as antibody. In view of the specificity of the antibody, the present results seem to suggest an increase in amount of degraded dermatan sulfate.

In addition to the formation of mechanical blisters, the characteristic feature of P-D-DEB is the development of small, firm, ivory-white papules, which enlarge slowly to an approximate diameter of $10 \mathrm{~mm}$. These papules are independent of the blister formation and are most frequent on the trunk, especially in its lumbosacral region. The other features of this disease are compatible with those of the CockayneTouraine form. As to the pathogenesis of $\mathrm{EB}$, numerous hypotheses have been put forward. Recent advances in molecular biology have contributed greatly to the elucidation of the etiopathogenesis of EB. In DEB, it seems likely that molecular abnormalities of type VII collagen participate in the pathogenesis of the disorder (Bruckner-Tuderman, 1992). Bauer et al. (1979) demonstrated an increased amount of GAGs in fibroblast cultures of seven patients with P-D-DEB, 
suggesting an abnormal catabolism of GAG in this disease. Recently, Priestley (1991) failed to show any unusual features in cell lines from six patients with P-D-DEB, as compared with six fibroblast lines from patients with the Cockayne-Touraine form, and brought into question the validity of P-D-DEB. Albo-papuloid lesions have been reported to occur in other forms of EB by a number of investigators (Kero, 1984; Tidman and Eady, 1985; Bavinck et al. 1987). The question as to whether P-DDEB and Cockayne-Touraine form represent different expressions of an identical mutant gene, or represent expressions related to different genes has not been resolved yet. Further advances in genetic analysis of EB are needed and these could provide definite solution of such as problem in near future.

\section{References}

Avnur, Z. and Geiger, B. (1984). Immunohistochemical localization of native chondroitin-sulfate in tissues and cultured cells using specific monoclonal antibody. Cell 38, 811-822.

Bauer, E.A., Fieler, W.K. and Esterly, N.B. (1979). Increased glycosaminoglycan accumulation as a genetic characteristic in cell cultures of one variety of dominant dystrophic epidermolysis bullosa. J. Clin. Invest. 64, 32-39.

Bavinck, J.N.B., van Haeringen, D., Ruiter, D. and VAN DER SCHROEFF, J.D. (1987). Autosomal dominant epidermolysis bullosa dystrophica: are the Cockayne-Touraine, the Pasini and the Bart-types different expressions of the same mutant gene? Clin. Genet. 31, 416-424.

Bruckner-Tuderman, L. (1992). Pathogenesis of mechanobullous disorders. Exp. Dermatol.
1, 115-120.

Couchman, J.R., Caterson, B., Christner, J.E. and BAKER, J.R. (1984). Mapping monoclonal antibody detection of glycosaminoglycans in connective tissues. Nature 307, 650-652.

Culling, C.F.A., Reid, P.E., Clay, M.G. and Dunn, W.L. (1974). The histochemical demonstration of o-acylated sialic acid in gastrointestinal mucins: their associations with the potassium hydroxide-periodic acid-Schiff effect. J. Histochem. Cytochem. 22, 826-831.

Endo, M., Yamamoto, R., Yoshizawa, Z., Sasai, Y. and SAITO, N. (1974). Urinary chondroitin of epidermolysis bullosa dystrophica et albopapuloidea (Pasini). Clin. Chim. Acta 67, 249-253.

Fine, J.-D., Bauer, E.A., Briggaman, R.A., Eady, R.A.J., Esterly, N.B. et al. (1991). Revised clinical and laboratory criteria for subtypes of inherited epidermolysis bullosa. A consensus report by the Subcomittee on Diagnosis and Classification of the National Epidermolysis Bullosa Registry. J. Am. Acad. Dermatol. 24, 119-135.

Hascall, V.C. and Hascall, G.K. (1981). Proteoglycans. In Cell Biology of the Extracellular Matrix, ed. Hay, E.D., pp. 39-63. New York: Plenum Press.

KERo, M. (1984). Occurrence of epidermolysis bullosa in Finland. Acta Dermatovenereol. 64, 57-62.

LiLuE, R.D. (1965). Histopathologic Technic and Practical Histochemistry, 3rd edn., New York: McGraw-Hill.

Poole, A.R., Weber, C., Pidoux, I., Chol, H. and Rosenberg, L.C. (1986). Localization of a dermatan sulfate proteoglycan (DS-PG II) in cartilage and the presence of an immunologically related species in other tissues. J. Histochem. Cytochem. 34, 619625.

PRiestley, G.C. (1991). Glycosaminoglycans production by cultured skin fibroblasts from the Pasini and Cockayne-Touraine forms of dominant dystrophic epidermolysis bullosa. J. Invest. Dermatol. 96, 
168-171.

Ripellino, J.A., Klinger, M.M., Margolis, R.U. and Margolis, R.K. (1985). The hyaluronic acid binding region as a soecific probe for the localization of hyaluronic acid in tissue sections. J. Histochem. Cytochem. 33, 1060-1066.

SASAI, Y. (1971a). Effect of tissue fixation on the histochemical identification of acid mucopolysaccharides. Tohoku J. Exp. Med. 104, 85-91.

SASAI, Y. (1971b). Identification of individual acid mucopolysaccharide in tissue sections. Tohoku J. Exp. Med. 105, 101-110.

SASAI, Y., SAIto, N. and SeiJI, M. (1973). Epidermolysis bullosa dystrophica et albopapuloidea. Arch. Dermatol. 108, 554-557.

ScotT, J.E. and Doruing, J. (1965). Differential staining of acid glycosaminoglycans (mucopolysaccharides) by Alcian Blue in salt solution. Histochemie 5, 221-233.

Sobue, M., Nakashima, N., Fukatsu, T., Nagasaka, T., Fukata, S. et al. (1989). Production and immunohistochemical characterization of a monoclonal antibody raised to proteo- glycan purified from a yolk sac tumor. Histochem. J. 21, 455-459.

Sobue, M., Nakashima, N., Fukatsu, T., Nagasaka, T., КатоH, T. et al. (1988). Production and characterization of monoclonal antibody to dermatan sulfate proteoglycan. J. Histochem. Cytochem. 36, 479-485.

TEngBlad, A. (1979). Affinity chromatography on immobilized hyaluronate and its application to the isolation of hyaluronate binding proteins from cartilage. Biochim. Biophys. Acta 578, 281-289.

Tidman, M.J. and EADY, R.A.J. (1985). Evaluation of anchoring fibrils and other components of the dermoepidermal junction in dystrophic epidermolysis bullosa by a quantitative ultrastructural technique. J. Invest. Dermatol. 84, 374-377.

Voss, B., Glossl, J., Cully, Z. and Kresse, H. (1986). Immunocytochemical investigation on the distribution of small chondroitin sulfate-dermatan sulfate proteoglycan in the human. J. Histochem. Cytochem. 34, 1013-1019. 